\title{
The first VEGAS-free, exact, 5D, polarized photon-to-e+e-pair conversion event generator
}

\author{
Denis Bernard* \\ LLR, Ecole polytechnique, 91128 Palaiseau, France \\ E-mail: denis.bernardein2p3.fr
}

\begin{abstract}
Current pair-conversion event generators, e.g. in Geant4, have been developed mainly for the simulation of electromagnetic showers in calorimeters. They don't sample the five-dimensional differential cross-section (5D DCS), but a product of 1D DCSs. Most of them use high-energy and/or small angle approximations. Also the e+ and e- polar angles are generated independently so energy-momentum is not conserved. None of them can simulate the conversion of polarized photons correctly.

In the past I wrote a generator that is sampling exactly the 5D Bethe-Heitler DCS. I used the VEGAS method: at a given energy, for a given target nucleus, after a 5D grid has been optimized, the DCS is tabulated, something that needs several seconds. Then zillions of conversions can be generated quickly at that energy and for that target. That software has undergone an extensive validation campaign. With an appropriate definition of the event azimuthal angle it was shown that the polarization asymmetry of the conversion agrees nicely with the low- and high-energy known asymptotic expressions.
\end{abstract}

I have developed a VEGAS-free version that allows the fast generation of the conversion of a photon of a given energy on a given target, with the same other properties as for the VEGASbased generator.

7th Fermi Symposium 2017

15-20 October 2017

Garmisch-Partenkirchen, Germany

${ }^{*}$ Speaker. 
This work originated from a theoretical and experimental development of low density, homogeneous detectors such as a gas time-projection chamber as a high-performance $\gamma$-ray telescope and polarimeter in the $\gamma \rightarrow e^{+} e^{-}$regime, that is, above $1 \mathrm{MeV}$ [1]. In contrast with past and current pair telescopes, the single-track angular resolution of such detectors can be so good that

- at low energy, the single-photon angular resolution is now dominated by the contribution of the momentum of the recoiling ion, that escapes measurement [2];

- polarimetry has been predicted to be possible despite the dilution of the polarization asymmetry induced by multiple scattering [3] and has actually been demonstrated by the characterization of a TPC prototype on beam $[4,5]$.

Therefore the simulation of modern, high-performance telescopes requires the use of an exact event generator,

- that describes correctly the recoil momentum distribution and

- that is able to simulate the conversion of linearly polarized $\gamma$ rays.

In addition, polarimetry is extremely demanding in terms of sample statistics, and the ability of gas detectors to detect low energy photons (see Fig. 1 of Ref. [6]) close to threshold $\left(2 m_{e} c^{2}\right.$ for "nuclear" conversion, i.e. in the field of a nucleus) is critical given the strongly decreasing dependence of the flux of cosmic sources with energy: for a $\Gamma=2$ spectral index source, the $\gamma \rightarrow e^{+} e^{-}$signal peaks at a couple of MeV (Fig. 2 of Ref. [3]). A generator that is free from high-energy approximation is needed. Last, upon conversion to a pair the final state is defined by a set of five variables (see Fig. 3 of Ref. [3]) and given the strong correlation between them in the differential cross section [3], the use of a generator that samples the 5D probability density function (pdf) is highly desirable.

Two frameworks are available for the simulation of high-energy physics (HEP) detectors and in particular of $\gamma$-ray telescopes: Geant4 [7, 8] and EGS5 [9]. Their $\gamma$-ray conversion generators have been developed so as to reproduce the properties of electromagnetic showers as precisely as possible, not for the simulation of high-performance $\gamma$-ray telescopes. None of these frameworks include an event generator that samples the 5D pdf: instead products of 1D pdfs are used. Most often, the electron and the positron are generated back-to-back and their polar angles are generated independently: there happens to be some recoil induced from the momentum mismatch then, that is bound to lie in the conversion plane, but that has nothing to do with the true, QED, recoil momentum distribution [10]. Energy-momentum is obviously not conserved by these generators. The polarized physics model available in Geant 4 does not simulate the conversion of linearly polarized $\gamma$-rays to pairs at low energy where it is most needed; at higher energy it provides an erratic value of the polarization asymmetry (Fig. 11 of [10]).

I have written an event generator that samples exactly (no high-energy nor small-angle approximation) the 5D differential cross section, including for photons with a non-zero linear polarization fraction [3]. This event generator is using the BASES/SPRING instantiation [11] of the VEGAS method [12]. The differential cross section can be computed by either of two methods:

- by the computation of all Feynman diagrams (See Fig. 3 of Ref. [3]) that contribute to first order of the Born approximation, using the HELAS software [13]; 
- or using the analytical expression obtained by Bethe and Heitler in the unpolarized case $[14,15]$ from the computation of the two diagrams that dominate nuclear conversion, and by $[16,17,18]$ in the fully polarized case (complete expressions, including for partially polarized photons, can be found in our [19]).

These two methods (HELAS, Bethe-Heitler) were extensively compared to each other, which proved to be extremely useful, in particular during the debugging phase of the project. The initial publication of the generator contained a number of validations [3]:

- The value of the most probable value of the opening angle was compared to the analytical calculation by Olsen [20] (Fig. 4 of Ref. [3]);

- The ratio of the recoil momentum distribution for triplet to nuclear conversions for various values of the energy of the incident photon was compared to the analytical calculation by Mork [21] (Fig. 5 of Ref. [3]);

- The variation of the triplet cross section $\sigma\left(q>q_{0}\right)$ above a recoil momentum threshold, $q_{0}$, as a function of $q_{0}$ for several energies was compared with the high-energy asymptotic expression of Ref. [22] (Fig. 6 of Ref. [3]);

- The total cross section for triplet conversion was compared to the computation by Mork [21] (Fig. 28, supplementary data of Ref. [3]);

- The decrease of the total mass attenuation coefficients when screening is applied (coherent form factor for nuclear conversion [23], incoherent form factor for triplet conversion [24]) was compared with calculations by Hubbell [25] (Fig. 29, supplementary data of Ref. [3]);

Further results were obtained recently:

- Validation of the value of the polarisation asymmetry, compared to the analytical expressions of the high-energy (eq. (14) of Ref.[19]) and low-energy asymptote (eq. (16) of Ref.[19]) of the polarisation asymmetry (Fig. 1).

- Comparison of $q_{X}$, the value of the recoil momentum $q$ at $X \%$ containment, for three values of $X$ (Fig. 2).

- Comparison of the distributions of $x_{+}=E_{+} / E_{\gamma}$ and $\theta_{+-}$for the HELAS and BH models (Fig. 2).

Using the VEGAS method, at a given energy, for a given target nucleus (nuclear conversion) or atom (triplet conversion), after a 5D grid has been optimized for differential cross section integration precision, the differential cross section is tabulated and stored, something that needs several seconds of computation. Then zillions of $\gamma$ conversions can be generated quickly at that energy and for that target.

I have developed recently a VEGAS-free version of that code, that allows the fast generation of the conversion of a photon of a given energy on a given target, with the same other properties as for the VEGAS-based generator, i.e., 5D, exact, polarized and strictly energy-momentum conserving. I have extended the validation energy range down to $1.024 \mathrm{MeV}$ and up to $100 \mathrm{PeV}$. A C++ version of that software is being prepared so as to attempt integration into Geant 4 as a new physics model. 

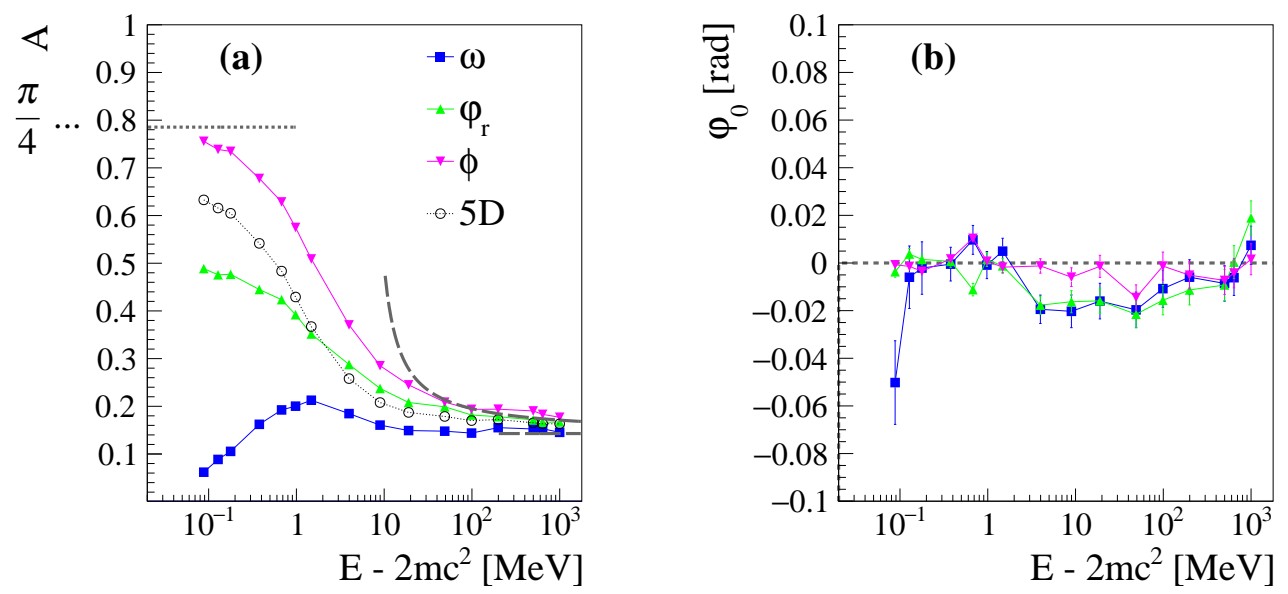

Figure 1: Amplitude $A$ (left) and phase $\varphi_{0}$ (right) of the modulation of the distribution for various definitions of the azimuthal angle [19] (squares: $\omega$, upward triangles: $\varphi_{r}$, downward triangles: $\phi$. In addition, the performance of the 5D estimator is shown (circles). The dotted lines show: (a) the asymptotic values of $A=\pi / 4$ at low energy. The dashed curve shows the high energy asymptotic expression for $A$ (eq. (16) of Ref.[19]) (a). These results were obtained using simulated samples with $P=1$ and $N=10^{5}$ events each. Please note that the same quantity (example $A$ ) is measured for various definitions of the azimuthal angle using the same event sample at a given photon energy, so that their statistical fluctuations are correlated. The error bars in plot (a) amount to $\approx \sqrt{2 / N} \approx 0.0045$ and are therefore not visible.
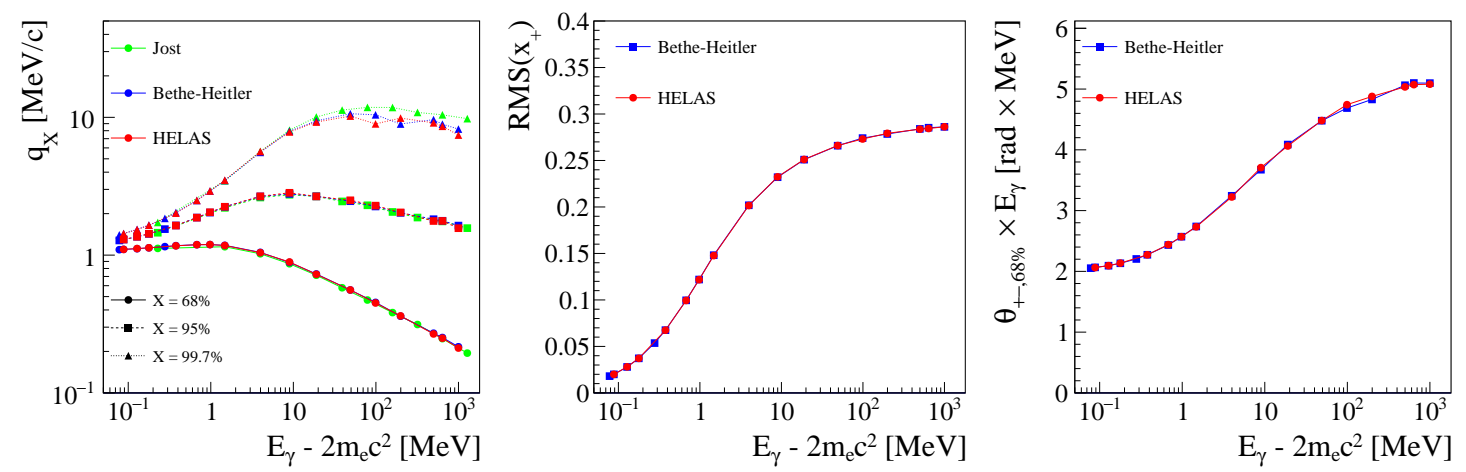

Figure 2: Left: The containment value $q_{X}$ as a function of the photon energy, for different values of the percentile $X$ [10]. Comparison of the variables $x$ (center) and $\theta_{+-}$(right) between BH and HELAS [10].

It is a pleasure to acknowledge the support of the French National Research Agency (ANR13-BS05-0002).

\section{References}

[1] HARPO, "hermetic argon polarimeter",

http://llr.in2p3.fr/ dbernard/polar/harpo-t-p.html

[2] D. Bernard, "TPC in gamma-ray astronomy above pair-creation threshold," Nucl. Instrum. Meth. A 701, 225

(2013) Erratum: [Nucl. Instrum. Meth. A 713, 76 (2013)]. [arXiv:1211.1534 [astro-ph.IM]]. 
[3] D. Bernard, "Polarimetry of cosmic gamma-ray sources above $e^{+} e^{-}$pair creation threshold", Nucl. Instrum. Meth. A 729 (2013) 765. [arXiv:1307.3892 [astro-ph.IM]].

[4] P. Gros et al., "Measurement of polarisation asymmetry for gamma rays between 1.7 to $74 \mathrm{MeV}$ with the HARPO TPC”, SPIE2016, 9905-95, arXiv:1606.09417 [astro-ph.IM].

[5] P. Gros et al., "Performance measurement of HARPO: a Time Projection Chamber as a gamma-ray telescope and polarimeter," arXiv:1706.06483 [astro-ph.IM], to appear in Astroparticle Physics, doi 10.1016/j.astropartphys.2017.10.008

[6] D. Bernard, "HARPO: 1.7 - $74 \mathrm{MeV}$ gamma-ray beam validation of a high angular resolutio n, high linear polarisation dilution, gas time projection chamber telescope and polarimeter," presented at SciNeGHE2016, October 2016, Pisa, Italy, Il Nuovo Cimento 40 C (2017) 117, arXiv:1702.08429 [astro-ph.IM].

[7] S. Agostinelli et al. [GEANT4 Collaboration], "GEANT4: A Simulation toolkit,” Nucl. Instrum. Meth. A 506, $250(2003)$

[8] M. Asai et al. [Geant4 Collaboration], "Recent developments in Geant4," Annals Nucl. Energy 82 (2015) 19.

[9] H. Hirayama et al., "The EGS5 code system," SLAC-R-730, KEK-2005-8, KEK-REPORT-2005-8, version: January 13, 2016.

[10] P. Gros and D. Bernard, " $\gamma$-ray telescopes using conversions to $e^{+} e^{-}$pairs: event generators, angular resolution and polarimetry," Astropart. Phys. 88 (2017) 60 [arXiv:1612.06239 [astro-ph.IM]].

[11] S. Kawabata, "A New version of the multidimensional integration and event generation package BASES/SPRING,” Comput. Phys. Commun. 88 (1995) 309.

[12] G. P. Lepage, “A New Algorithm for Adaptive Multidimensional Integration,” J. Comput. Phys. 27 (1978) 192.

[13] H. Murayama, I. Watanabe and K. Hagiwara, "HELAS: HELicity amplitude subroutines for Feynman diagram evaluations," KEK-91-11.

[14] H. Bethe \& W. Heitler, "On the Stopping of Fast Particles and on the Creation of Positive Electrons", Proceedings of the Royal Society of London A, 146 (1934) 83.

[15] H. A. Bethe, "The influence of screening on the creation and stopping of electrons", Mathematical Proceedings of the Cambridge Philosophical Society, 30 (1934) 524.

[16] T. H. Berlin and L. Madansky, "On the Detection of gamma-Ray Polarization by Pair Production", Phys. Rev. 78 (1950) 623

[17] M. M. May, "On the Polarization of High Energy Bremsstrahlung and of High Energy Pairs”, Phys. Rev. 84 (1951) 265

[18] Jauch and Rohrlich, "The theory of photons and electrons", (Springer Verlag, 1976).

[19] P. Gros and D. Bernard, " $\gamma$-ray polarimetry with conversions to $e^{+} e^{-}$pairs: polarization asymmetry and the way to measure it,” Astropart. Phys. 88 (2017) 30 [arXiv:1611.05179 [astro-ph.IM]].

[20] H. Olsen, “Opening Angles of Electron-Positron Pairs,” Phys. Rev. 131 (1963) 406.

[21] K. J. Mork, "Pair Production by Photons on Electrons", Phys. Rev. 160 (1967) 1065.

[22] M. L. Iparraguirre and G. O. Depaola, "Pair production by gamma-rays on electrons. Threshold for the momentum recoil detection,” Eur. Phys. J. C 71 (2011) 1778.

[23] N.F. Mott, H.S.W. Massey, “The Theory of Atomic Collisions”, University Press, Oxford, 1934.

[24] J.A. Wheeler and W.E. Lamb, “Influence of atomic electrons on radiation and pair production", Phys. Rev. 55 (1939) 858 (errata in 101 (1956) 1836).

[25] J. H. Hubbell et al., "Pair, Triplet, and Total Atomic Cross Sections", J. Phys. Chem. Ref. Data 9 (1980) 1023. 